\title{
PROPOSAL FOR A MULTI-USE TEST BEAM IN THE SLAC B-LINE *
}

\author{
P. Emma ${ }^{\dagger}$, L. Bentson, R. Erickson, T. Fieguth, J. Seeman, A. Seryi (SLAC)
}

\section{Abstract}

With the impending construction of the Linac Coherent Light Source (LCLS) [1] at SLAC, displacing the wellused Final Focus Test Beam (FFTB) area, there is growing interest in developing a new test beam facility which makes use of the remaining 2/3 of the SLAC linac, and is available during LCLS operations. The success of the Sub-Picosecond Pulse Source (SPPS) [2] and the desire to preserve this capacity suggest a new beamline with similar or improved electron beam quality, including bunch length compression to $10 \mu \mathrm{m}$. Beam availability during LCLS operations requires a new 1-km bypass beamline connecting the 2/3-point of the linac with, for example, the existing B-Line tunnel at the end of the linac. A second operating mode, with LCLS not running, is then available using the existing connection directly from the end of the linac to the B-line. This path would provide the highest beam quality at $30 \mathrm{GeV}$ and also allow a third operational mode by deflecting a few of the very high-brightness $120-\mathrm{Hz}, 14-\mathrm{GeV}$ LCLS bunches at low rate $(1-10 \mathrm{~Hz})$ into the B-line. Additionally, linear collider research might also be carried out in a short final focus system at the end of the B-Line, capable of producing a 70-nm rms transverse beam size. We describe a design for these systems.

\section{THE BYPASS LINE}

The 3-km long SLAC linac is composed of 30 equal length sectors, each about $100 \mathrm{~m}$ in length. The LCLS injector will be located at the start of sector-21 (the 2/3-point) and will begin full operations in 2009, using about $75 \%$ of available linac time. With PEP-II presently using the first $2 / 3$ of the linac and running through 2008 , there is growing interest in developing a new test beam facility which makes use of this 2-km linac in parallel with LCLS operations. This requires a 1-km long linac-bypass line starting in sector-20 and running along the tunnel ceiling to the linac end. Similar bypass lines are used for a positron return line, and the $3-\mathrm{GeV}$ positron and $9-\mathrm{GeV}$ electron transport lines to the PEP-II ring. Space, although tight, does exist on the ceiling for a new line from sector-20 and beyond. The new line can operate with DC bends, since the LCLS injector is downstream of the sector-20 take-off point.

The new bypass line described here begins at sector 20-5 with a pair of 20-mrad angle bends forming a linear achromat of 65-m length. The bend magnets are rolled 23 degrees to place the bypass line on the ceiling $1.14 \mathrm{~m}$ horizontally (south) and $0.48 \mathrm{~m}$ vertically (up) from the linac axis.

\footnotetext{
* Work supported by US Department of Energy contract DE-AC02$76 \mathrm{SF} 00515$.

†Emma@SLAC.Stanford.edu
}

Eight quadrupole mirror-plate magnets between the bend pairs form an achromat and limit the first and second-order compression factors to $\left|R_{56}\right| \approx 0.3 \mathrm{~mm}$ and $\left|T_{566}\right| \approx 56$ $\mathrm{mm}$, respectively. This preserves the short bunch length (50 $\mu \mathrm{m} \mathrm{rms)} \mathrm{generated} \mathrm{in} \mathrm{the} \mathrm{sector-10} \mathrm{chicane} \mathrm{[3].}$

The bypass line itself is a simple 45-deg/cell FODO lattice with 10 quadrupoles over a 1-km length. Four independently adjustable matching quadrupoles are used both before and after the FODO line.

Near the end of the linac, the beam is bent back down and to the left (see Fig. 1), towards the linac axis with a 12mrad pair of bends (BY3, BY4), each rolled at 42 degrees, through six quadrupoles, placing the beam on the nominal B-Line axis a few meters upstream of the existing D-10 dump box. A third unrolled bend (BY5) sets the right-bend angle to 0.5 degrees, for launch into the B-Line. The vertical dispersion is closed by BY4, whereas the BS1 bend closes the horizontal dispersion. With this arrangement the BY4 and BY5 magnets (see Fig. 1) can be switched off and the linac beam (without bypass) taken directly into the BLine using the existing B1 and B2 bends when LCLS is not running. Similarly, the existing pulsed bends near B1 and B2 (not shown) can be used to steal 1-10 Hz LCLS pulses directly into the B-line, allowing three different operational modes.

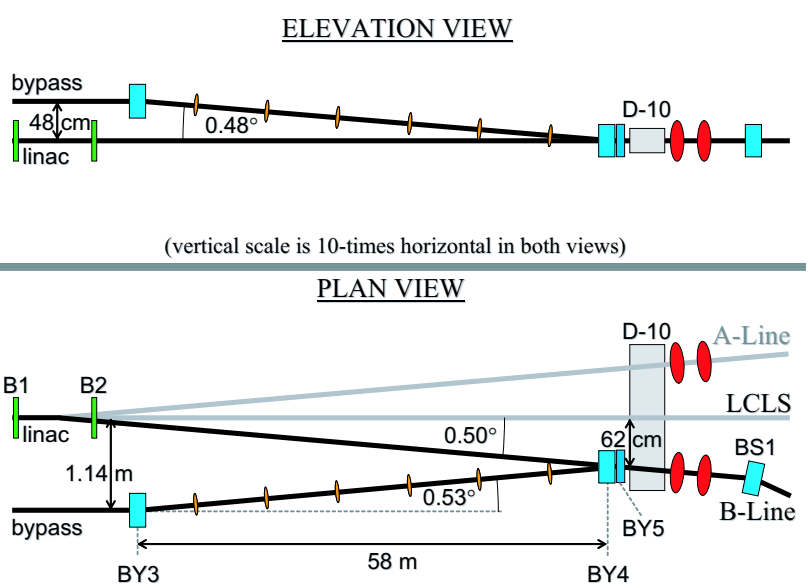

Figure 1: Bypass line and linac connection into and through the start of the B-Line, shown in elevation and plan view.

\section{THE B-LINE}

The B-Line is an original feature of the SLAC complex, branching off the end of the linac at 12.5 degrees towards the south, extending about $150 \mathrm{~m}$ in length. The spacious, well built enclosure has been unused for at least 20 years 
and many of the original components have been removed. The tunnel ends in a wide B-Line Target Room (BTR), with a convenient separate access tunnel, followed by the large "End Station B" (ESB) hall, having plenty of room for experimental apparatus. A large shielding wall separates the target room from the linac, allowing access during linac operations.

A new beamline optics has been designed, following the existing tunnel and allowing beam energies up to 30 $\mathrm{GeV}$. The optics provides bunch compression similar to the FFTB with the SPPS beam, with an adjustable linear compression factor of $R_{56} \approx 2.3 \mathrm{~mm}$. The optics is shown in Fig. 2, where the first dipole (at $s \approx 248 \mathrm{~m}$ ) is the BS1 bend shown in Fig. 1. In addition, an emittance diagnostic section, with optimal phase advance, is included at $s \approx 268-294 \mathrm{~m}$, where three OTR screens or wire scanners can be located to measure the horizontal and vertical emittance. The four final 1.5-degree bends at $s \approx 302-328$ $\mathrm{m}$ are placed to follow the tunnel. A few meters long undulator, such as that used in the FFTB/SPPS, can be located after this section to produce $1-\AA$ spontaneous $\mathrm{X}$-ray radiation to continue SPPS femtosecond $\mathrm{x}$-ray research.

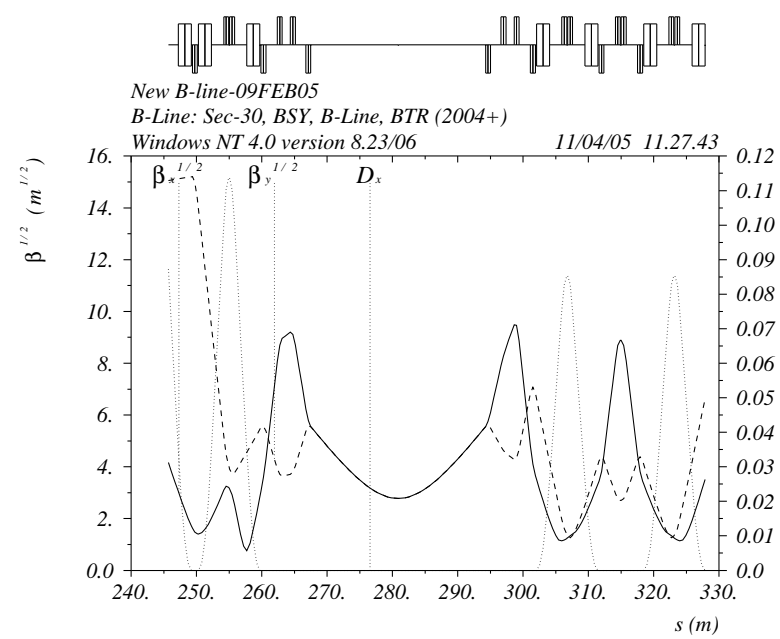

Figure 2: New B-Line optics starting at BS1 dipole and extending to the pre-target room shield wall.

Particle tracking through the B-Line with LiTrack [4] in 2D, via linac or bypass line, is shown in Fig. 3, producing 11 or $24 \mathrm{kA}$ of peak current with $3.4 \mathrm{nC}$ of bunch charge at $28.5 \mathrm{GeV}$. The LCLS beam (not shown), with pulsed RF phases, can generate even shorter bunches, to a few microns length and up to $40-50 \mathrm{kA}$, but with less charge (1 $\mathrm{nC})$.

Most of the required magnets, except the bypass line quadrupoles, are available from the existing and obsolete SLC final focus systems. The bypass line quadrupoles can be taken from existing designs. Only the eight low-profile, mirror plate quadrupoles will need to be designed and built.
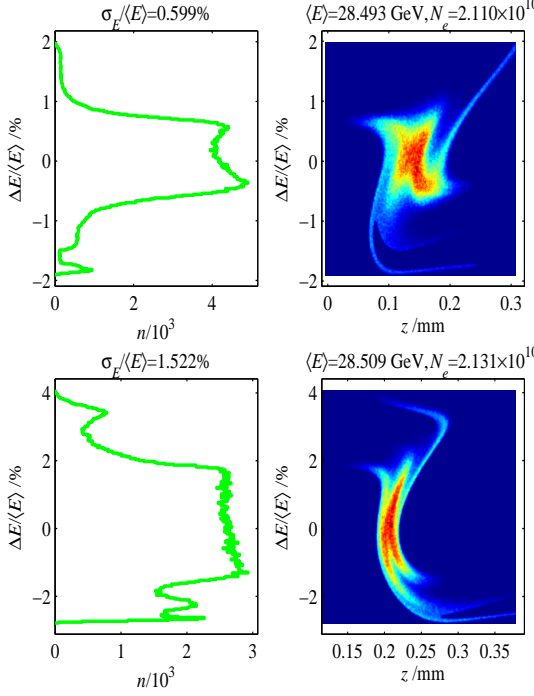
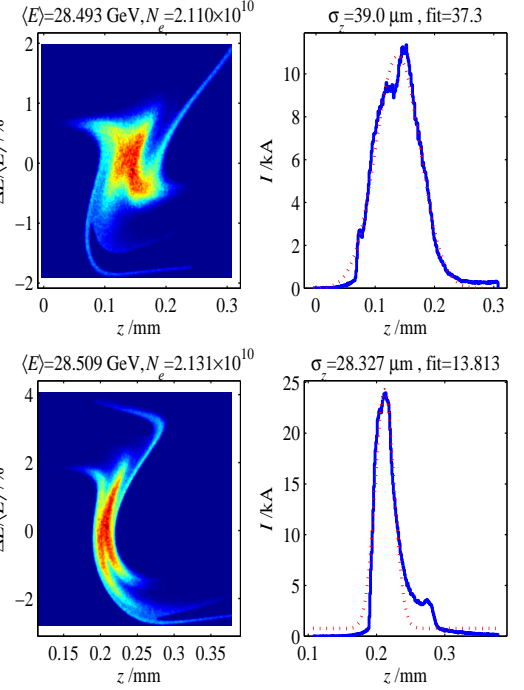

Figure 3: Energy profile (left), longitudinal phase space (center), and longitudinal profile (right) after B-line, through bypass line (top), and through full linac (bottom).

\section{FINAL FOCUS OPTICS}

A further option is to include a final focus system at the end of the new B-Line, starting at the $s \approx 330$-m point in Fig. 2. The optics for such a scheme is based on the NLC Final Focus (now adopted for ILC) originally suggested in Ref. [5]. Compensation of chromaticity is performed locally by sextupoles inserted in the final doublet (FD), where the dispersion is nonzero. The second order dispersion created in the FD is compensated simultaneously with vertical and horizontal chromaticity by allowing half of the horizontal chromaticity to arrive from upstream, doubling the amount produced by the FD quadrupoles. Compensation of geometrical aberrations is achieved with sextupoles located upstream. In comparison with the traditional final focus optics with non-local chromaticity compensation, such optics provides much better performance in a shorter system.

The B-Line FF optics was scaled from the latest NLC FF design described in [6]. The distance from the focus to the first quadrupole $\left(L^{*}\right)$ is chosen to be $1 \mathrm{~m}$ and the design beta functions at the focus are $\beta_{x}=4 \mathrm{~mm}, \beta_{y}=0.1 \mathrm{~mm}$; thus, this optics has about the same vertical chromaticity as the ILC FF (which has four times larger $L^{*}$ and $\beta_{y}$ ). In order to be compatible with short bunch operation, the optimization procedure of the FF optics [7] was augmented by the additional constraints to zero the $R_{56}$ and to limit the $T_{566}$ terms of the transfer matrix.

The FF optics is shown in Fig. 4. The length of the FF system was defined by the available space in the B-Line. In the present design, the distance from the focus to the iron wall which separates the target room from the ESB experimental hall is about $23 \mathrm{~m}$, which should be sufficient to accommodate the beam dump and possible x-ray instrumentation. The optics provides about four drifts near the beginning of the FF with combined length of about $6 \mathrm{~m}$ 


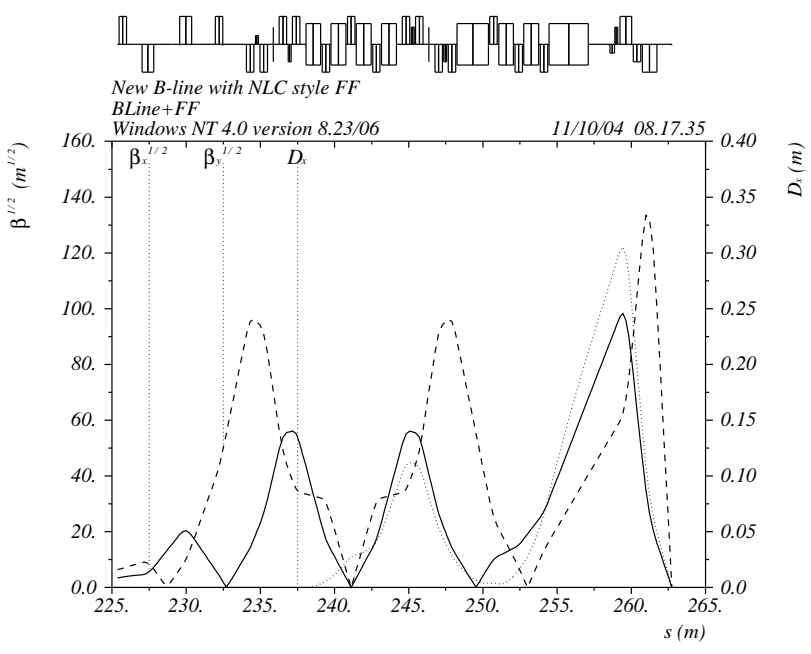

Figure 4: Final focus optics for the new B-Line. (The $s$ coordinate here is shifted $\sim 100$ m relative to Fig. 2.)

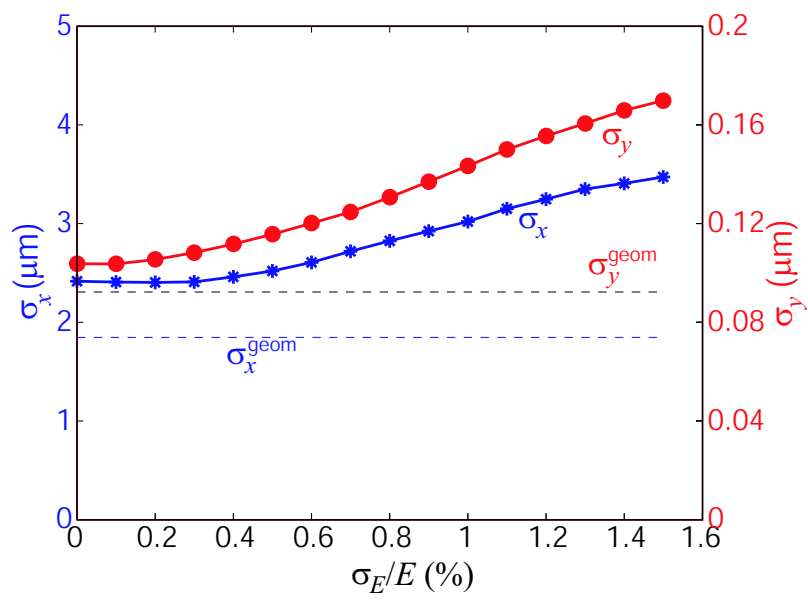

Figure 5: Bandwidth of the B-Line with FF optics for 'SPPS-like' beam.

that might also be used to accommodate undulators or other diagnostics. Adding the FF system requires splitting the existing shield wall into two $1.5-\mathrm{m}$ sections and moving it several meters upstream. The strengths of the FF magnets, and in particular the FD, are sufficient to focus the electron beam up to $30 \mathrm{GeV}$.

The energy bandwidth of the B-Line with FF optics for the 'SPPS-like' beam is shown in Fig. 5. Due to the large emittance of this damping-ring-generated beam, there is a noticeable increase of the beam size due to geometric and chromatic aberrations, indicating that the IP beta functions might be too aggressive for such a beam. For the LCLS beam, with its much smaller emittance and energy spread, these aberrations play a negligible effect and the tracked beam size is very close to the design (linear) value.

The B-Line final focus optics is compatible with short bunch operation: the FF optics has little effect on the linear compression term $\left(\left|\Delta R_{56}\right| \approx 0.1 \mathrm{~mm}\right)$ but doubles the second order term (the total value with FF optics
$\left|T_{566}\right| \approx 77 \mathrm{~mm}$ ), which is acceptable. A realistic energy distribution, tracked through the linac wakefields, was used in this case. The parameters of the B-Line with FF optics, in terms of achievable bunch length and transverse beam size at the focus (including synchrotron radiation), are summarized in Table 1.

Table 1: B-line parameters with FF system for three modes of operation, including synchrotron radiation.

\begin{tabular}{|l|c|c|c|r|}
\hline param. & bypass & SPPS-like & LCLS & unit \\
\hline$E_{0}$ & 30 & 30 & 14 & $\mathrm{GeV}$ \\
$Q$ & 3.4 & 3.4 & 1.0 & $\mathrm{nC}$ \\
$\gamma \varepsilon_{x(y) 0}$ & $75(10)$ & $50(5)$ & $1.0(1.0)$ & $\mu \mathrm{m}$ \\
$\sigma_{E} / E_{0}$ & 0.60 & 1.5 & $<0.1$ & $\%$ \\
$\sigma_{z}^{\text {eff }}$ & 37 & 14 & $3-30$ & $\mu \mathrm{m}$ \\
$I_{p k}$ & 11 & 24 & $40-4$ & $\mathrm{kA}$ \\
$\beta_{x(y)}^{*} \mathrm{IP}$ & $4.0(0.10)$ & $4.0(0.10)$ & $4.0(0.10)$ & $\mathrm{mm}$ \\
$\sigma_{x(y)}^{*}$ & $4.5(0.24)$ & $4.6(0.18)$ & $0.53(0.07)$ & $\mu \mathrm{m}$ \\
\hline
\end{tabular}

The beam dump is not yet designed. Such a system will likely be composed of permanent-magnet vertical dipoles deflecting the beam safely into a high-power, underground dump, possibly located at the end of the B-target room. Detailed tracking and tolerance studies remain to be done for the full set of beamlines.

\section{OTHER TEST BEAMS}

Throughout SLAC's history, beams have been used for calibration of particle detectors and testing of detector prototypes. For a large portion of these experiments, a medium energy, low flux beam is ideal. Such a beam can be used directly or as input to an apparatus providing tagged photons. In the past, these beams have been provided in both the FFTB facility and ESA (End Station A), running parasitically to SLC. The proposed BTR beamline can conceivably provide this same capability while running parasitically to LCLS. If the proposed beamline includes $4-\mathrm{cm}$ aperture quadrupoles, it will have an acceptance comparable to the FFTB and ESA beamlines. Placing an appropriate target 1-meter upstream of the location of the existing hadron generation target, between $\mathrm{B} 1$ and $\mathrm{B} 2$, but off the axis of the LCLS beam, would allow the use of low flux, 7 $\mathrm{GeV} e^{ \pm}$beams in the BTR. Further studies of these rates and range of achievable momenta are warranted.

\section{REFERENCES}

[1] LCLS CDR, SLAC Report No. SLAC-R-593, 2002.

[2] P. Emma et al., PAC'01, Chicago, IL, June 18-22, 2001.

[3] P. Emma et al., EPAC'02, Paris, France, June 3-7, 2002.

[4] K.L.F. Bane and P. Emma, these PAC'05 proceedings.

[5] P. Raimondi and A. Seryi, Phys. Rev. Lett. 86, 3779 (2001).

[6] A. Seryi et al., EPAC’04, Paris, France, July 2004.

[7] A. Seryi et al., PAC'03, Portland, OR, May 2003. 